\title{
AVALIAÇÃO DA CONTAMINAÇÃO POR PARASITOS E COLIFORMES EM ALFACES (Lactuca sativa) COMERCIALIZADAS EM FEIRAS LIVRES NA CIDADE DE JEQUIÉ, BAHIA.
}

\author{
EVALUATION OF CONTAMINATION BY PARASITES AND COLIFORMS IN \\ LETTUCE (Lactuca sativa) SOLD AT STREET MARKETS IN THE CITY OF \\ JEQUIÉ, BAHIA.
}

Dellane Tigre, Yana Queiroz Rodrigues, Samilla Gonzaga Souza, Virgínia Maria Góes da Silva

Universidade Estadual do Sudoeste da Bahia - UESB

Abstract

In Brazil, more than half of the municipalities do not have basic sanitation plans. This situation affects, in fact, the most economically disadvantaged population, causing the onset of illness by eating raw or poorly washed foods. The present study aimed to analyze the presence of parasites, total and thermotolerant coliforms in lettuces (Lactuca sativa) sold in street markets in Jequié, Bahia. From August to October 2019, the samples were obtained randomly from three street markets, the analyzes were performed by the simple centrifugation method and coliform survey used the Most Probable Number Method. of the lettuce samples analyzed in street markets, $62.5 \%$ showed contamination by hookworm, being the predominant larvae morphology and, the egg was found in only one sample; in addition, it was found that $90.9 \%$ of the analyzed samples were positive for thermotolerant coliforms. The presence of hookworms as well as the high count of thermotolerant coliform in samples of lettuce obtained in street markets in the city of Jequié, indicates the need for implementation and adoption of prophylactic measures during the commercialization of vegetable.

Keywords: Parasite. Coliform. Lettuce. Food.
Resumo

No Brasil, mais da metade dos municípios não possuem plano de saneamento básico. Essa situação atinge, de fato, a população socioeconomicamente mais carente, ocasionando o aparecimento de doenças por ingestão de alimentos crus ou mal lavados. O presente estudo teve por objetivo analisar a presença de parasitos, coliformes totais e termotolerantes em alfaces (Lactuca sativa) comercializadas em feiras livres em Jequié, Bahia. Entre os meses de agosto a outubro de 2019, as amostras foram obtidas aleatoriamente em três feiras livre, as análises foram realizadas pelo método de centrifugação simples e a pesquisa de coliformes utilizou o Método do Número Mais Provável. As amostras de alface analisadas nas feiras livres, 62,5\% apresentaram contaminação por Ancilostomídeo, sendo a morfologia predominante larva e, em apenas uma amostra foi encontrado um ovo; além disso, foi possível constatar que 90,9\% das amostras analisadas foram positivas para coliformes termotolerantes. A presença de Ancilostomídeo assim como a elevada contagem de coliformes termotolerantes em amostras de alfaces obtidas em feiras livres no município de Jequié, sinaliza a necessidade da implementação $e$ adoção de medidas profiláticas durante o processo de comercialização das hortaliças.

Palavras-chave: Parasito. Coliformes. Alface. Alimentos. 
A alface (Lactuca sativa) é considerada a hortaliça mais consumida em todo o mundo, devido ao seu aporte nutricional é um alimento rico em fibras, minerais e vitaminas necessárias para o bom funcionamento do corpo humano ${ }^{1}$. Pesquisas apontam que o consumo de hortaliças cruas está diretamente relacionado com a alimentação saudável, entretanto, em diversas partes do mundo, o consumo desses vegetais pode estar relacionado a transmissão de patógenos ${ }^{2,3,4}$, principalmente quando associados as péssimas condições sanitárias ao qual o indivíduo está inserido.

No Brasil, mais da metade dos municípios não possuem plano de saneamento básico ${ }^{5}$. De fato, essa situação atinge, basicamente, a população socioeconomicamente mais carente, ocasionando o aparecimento de doenças por ingestão de alimentos crus ou mal lavados ${ }^{6,4}$. Segundo Ferreira e col. ${ }^{7}$, de 2007 a 2017 foram relatados no Brasil 131.285 casos de doenças ocasionadas pela ingestão de alimentos, destes, $1,08 \%$ indicaram as hortaliças como a causa do acontecimento. Das enfermidades parasitárias, as enteroparasitoses com rota orofecal são as mais prevalentes ${ }^{6}$.

A detecção de ovos ou larvas de Ascaris lumbricoides, Ancilostomídeos e Strongyloides sp. é comum em alfaces adquiridas em supermercados ou outros comércios do Brasil, como descrito nas regiões $\mathrm{Sul}^{8}$ e Nordeste ${ }^{9}$.

Análises microbiológicas realizadas com hortaliças coletadas em Campos de Goytacazes, Rio de Janeiro, identificaram que $83 \%$ das hortaliças $(n=60)$, estavam contaminadas por coliformes termotolerantes e $98 \%$ indicavam positividade para coliformes totais, todas as amostras apresentaram contagens acima daquelas permitidas pela legislação vigente ${ }^{10}$.

Todos esses fatores revelam a importância da implementação de ações de segurança que visem esclarecer os produtores e consumidores sobre a correta higienização do ambiente e do alimento que será consumido in natura. Sabendo-se que, as doenças transmitidas por alimentos constituem um dos principais problemas de saúde pública no Brasil, torna-se relevante a investigação da qualidade sanitária dos alimentos consumidos no país. Dessa forma o objetivo desse estudo foi pesquisar parasitos e a presença de coliformes totais e fecais em amostras de alfaces comercializadas em feiras livres no município de Jequié, Bahia.

\section{Amostras}

Entre os meses de agosto a outubro de 2019, foram coletadas 40 amostras de alface crespa, em diferentes feiras livres do município. Nas feiras identificadas como A, B e C foram coletadas amostras de alface, aleatoriamente, no período matutino. Nas feiras A e B coletou-se sete barracas e na feira $C$ selecionou-se em seis barracas. As amostras foram coletadas em duplicata e em dias diferentes. As hortaliças foram armazenadas em sacolas plásticas próprias para alimentos e encaminhadas, sob refrigeração, ao laboratório para as análises.

\section{Análise parasitológica das amostras de alface}

As amostras foram avaliadas pelo método de centrifugação simples ${ }^{9}$. Com a utilização de luvas, foram descartadas as partes deterioradas das folhas e pesadas 20 gramas de cada amostra da alface em um Becker. Em seguida, com as folhas cortadas, adicionou-se $250 \mathrm{~mL}$ de água e a hortaliça foi homogeneizada com o auxílio de um bastão de vidro. A solução da lavagem foi filtrada com uma peneira coberta por gaze para um cálice de sedimentação. Para decantação das estruturas parasitárias, a amostra permaneceu em repouso por 24 horas a $4^{\circ} \mathrm{C}$. Posteriormente, o sobrenadante foi descartado com cuidado, para não homogeneizar o sedimento, até restar aproximadamente $30 \mathrm{~mL}$. Foram transferidos 14 $\mathrm{mL}$ do fundo do cálice para tubos Falcon cônico e, em seguida centrifugados a $300 \mathrm{~g}$, por 10 minutos (Excelsa II Mod. 206 BL). Após esta etapa, o sobrenadante foi descartado e todo o restante do sedimento foi analisado em lâminas cobertas por lamínulas no microscópio óptico nas objetivas de $10 x$ e $40 x$.

Técnica dos Tubos Múltiplos para pesquisa de coliformes.

A técnica dos tubos múltiplos é baseada no método do número mais provável, preconizada em Silva, Junqueira e Silveira ${ }^{11}$. Para a determinação de coliformes totais e termotolerantes foram utilizados o Caldo Verde Brilhante Bile (VBB) e o Caldo Escherichia coli (EC), respectivamente. Alíquotas de $1,0 \mathrm{ml}$ das três diluições seriadas da amostra $\left(10^{-1}, 10^{-2}, 10^{-3}\right)$ em água peptonada estéril, foram semeadas nos meios de cultura e colocados em estufa à temperatura de $35 \circ \mathrm{C}$ por 24 e 48 horas. Logo após, uma alçada de cada tubo que apresentou 
crescimento com produção de gás foi transferida para os tubos de Caldo EC. As amostras foram incubadas por 24 horas sob a temperatura de 45,5 으.

\section{Análise estatística}

Os dados de contagem de parasitos foram agrupados e tratados para o estabelecimento de frequência simples e percentual. Para a comparação das médias dos parasitos encontrados entre as três feiras foi aplicado o teste de Kruskal-Wallis, usando o programa GraphPad Prism 5.0.

\section{Resultados}

No presente estudo, todas as feiras livres do município de Jequié apresentaram alfaces crespas (L. sativa) contaminadas por parasito. Das amostras de alface analisadas nas feiras, $62,5 \%$ apresentaram contaminação por Ancilostomídeo, sendo a larva o estágio evolutivo predominante e, em apenas uma amostra foi encontrado o ovo.

$\mathrm{Na}$ feira A, 71,4\% das amostras avaliadas estavam contaminadas com larvas de Ancilostomídeo. $O$ percentual de amostras positivas observado na feira $B$ foi igual ao da feira
A, a única diferença foi a detecção de um ovo de Ancilostomídeo em uma das amostras ao invés da forma larvária. A feira C apresentou índice inferior de contaminação quando comparado as anteriores, $41,7 \%$ das amostras de alface apresentaram larvas de Ancilostomídeo. Não houve diferença estatística significativa entre as médias de amostras positivas entre as feiras $A, B$ e C.

Após os dois dias de obtenção da alface nas mesmas barracas, observou-se um percentual de $100 \%$ de bancas positivas para a presença de Ancilostomídeo nas feiras A e B. Enquanto na primeira feira mais da metade das barracas apresentaram positividade apenas nas amostras obtidas na segunda coleta, na B foram observadas duas bancas com alfaces contaminadas na primeira coleta e mais duas na segunda. Já na feira $C$, das seis barracas analisadas, $66,7 \%$ foram positivas para presença parasitária. Esse índice foi menor do que 0 encontrado nas feiras $A$ e $B$, destacando que em apenas $16,7 \%$ o parasito foi identificado nas duas amostras e em metade das bancas somente no segundo dia de coleta. Dessa forma, o percentual de concordância positiva entre as duas amostras coletadas em dias diferentes nas mesmas barracas foi de $42,8 \%$ nas feiras A e B e $16,7 \%$ para a C (Tabela 1).

Tabela 1 - Identificação da contaminação por Ancilostomídeo em amostras de alface (L. sativa) obtidas em duas coletas (primeira e segunda) nas três feiras livres da cidade de Jequié, Bahia.

\begin{tabular}{|c|c|c|c|c|c|c|}
\hline \multicolumn{3}{|c|}{ BARRACAS } & \multicolumn{3}{|c|}{ AMOSTRAS } & \\
\hline & \multicolumn{2}{|l|}{ Feira A } & \multicolumn{2}{|l|}{ Feira B } & \multicolumn{2}{|l|}{ Feira C } \\
\hline & Primeira & Segunda & Primeira & Segunda & Primeira & Segunda \\
\hline 1 & 0 & 1 & 0 & 1 & 0 & 1 \\
\hline 2 & 0 & 1 & 0 & 1 & 1 & 1 \\
\hline 3 & 0 & 1 & 1 & 0 & 0 & 0 \\
\hline 4 & 0 & 1 & 1 & 1 & 0 & 0 \\
\hline 5 & 1 & 1 & 1 & 0 & 0 & 1 \\
\hline 6 & 1 & 1 & 1 & 1 & 0 & 1 \\
\hline 7 & 1 & 1 & 1 & 2 & - & - \\
\hline
\end{tabular}

Para a pesquisa de coliformes, foram analisadas 11 amostras de alfaces, em triplicata de acordo com o método dos Tubos múltiplos. Todas as amostras foram positivas para coliformes totais e fecais apresentando, entretanto, diferentes 
contagens, o que demonstra uma variação na quantidade de coliformes presente (Tabela 2).

Tabela 2 - Número mais provável por grama (NMP/g) de coliformes totais e fecais em amostras de alfaces comercializadas em feiras livres na cidade de Jequié, Bahia.

\begin{tabular}{lllll}
\hline NMP/g & $\begin{array}{l}\text { Coliformes } \\
\text { totais }\end{array}$ & $\begin{array}{l}\text { Coliformes } \\
\text { totais }(\%)\end{array}$ & $\begin{array}{l}\text { Coliformes } \\
\text { termotolerantes }\end{array}$ & $\begin{array}{l}\text { Coliformes } \\
\text { termotolerantes } \\
(\%)\end{array}$ \\
\hline 22400 & 9 & $81,8 \%$ & 8 & $72,7 \%$ \\
240 & 1 & $9,1 \%$ & 2 & $18.2 \%$ \\
$<3$ & 1 & $9,1 \%$ & 1 & $9.1 \%$ \\
\hline
\end{tabular}

Conforme demostrado na Tabela 2, a maior parte das amostras, 81,8\% apresentaram valores acima de $2.400 \mathrm{NMP} / \mathrm{g}$ e um total de 10 amostras $(90,9 \%)$ foram positivas para coliformes totais. Entretanto não há valores máximos estabelecidos na legislação para a quantidade de coliformes totais nos alimentos.

Quanto aos coliformes termotolerantes 72,7\% das amostras apresentaram contagens acima de 2400NMP/g e um total de 10 amostras positivas. A legislação, RDC no 12 de 2001 da Agência Nacional de Vigilância Sanitária (ANVISA) ${ }^{12}$ preconiza a quantidade máxima de $10^{2} \mathrm{NMP} / g$ de coliformes a $45^{\circ} \mathrm{C}$ em hortaliças in natura, os resultados encontrados evidenciaram a elevada frequência de amostras contaminadas por coliformes totais e termotolerantes.

Quanto a distribuição das amostras por feira, nas feiras B e C $100 \%$ das amostras foram positivas para coliforme totais e fecais, já na feira $A$ houve positividade de $75 \%$ para coliformes totais e termotolerantes.

\section{Discussão}

A análise da qualidade sanitária das hortaliças, assim como os potenciais riscos à saúde relacionados ao seu consumo in natura, tem sido alvo de estudo de diversos autores ${ }^{8,13,14}$. Segundo o que foi estabelecido pela resolução no 12 , de 1978 da Comissão Nacional de Normas e Padrões para Alimentos (CNNAPA) ${ }^{15}$, e na RDC no 12 de 2001 (ANVISA) $^{12}$ as hortaliças devem estar isentas de sujeira e estruturas parasitárias. Os resultados do presente estudo mostram que as amostras analisadas estão em desacordo com a resolução e inapropriadas para ingestão. Ademais, o resultado sinaliza que as práticas de cultivo das alfaces podem estar sendo realizadas em condições higiênicas sanitárias inadequadas.

O percentual observado nas análises parasitológicas foi cinco vezes superior ao verificado na cidade do Rio de Janeiro em amostras de alface de cultivo tradicional e hidropônico comercializadas em feiras livres, entretanto, além de Ancilostomídeo, os autores identificaram oocisto não esporulado de coccídio e cisto de Amebídeo ${ }^{16}$.

No presente estudo foi identificado Ancilostomídeo, entretanto a similaridade morfológica não permitiu a diferenciação por microscopia óptica do gênero e espécie das larvas e ovo. Estudos similares identificaram a presença desse helminto em amostras colhidas em supermercados, feiras e restaurantes do Brasil $^{17,18}$. Normura e col. ${ }^{19}$, identificaram $87,5 \%$ de amostras contaminadas com larvas de Ancilostomídeos em feiras e supermercados na cidade de Londrina, Paraná.

De acordo com Abougrain e col. ${ }^{2}$ o tipo de parasito identificado em hortaliças pode variar com o local de obtenção da amostra, seja área rural ou urbana, com os índices de saneamento básico e com a quantidade das amostras. A irrigação das hortaliças com água proveniente de rios e poços contaminados por esgotos ou fossas também pode determinar a variabilidade da espécie contaminante. Outro fato a ser considerado é a forma estrutural da alface variedade crespa, uma vez que suas folhas justapostas e largas dificultam a adesão dos parasitos $^{20}$. Além disso, o clima da região, quente, úmido ou temperado, pode favorecer a prevalência e predominância de determinados parasitos em detrimento de outros, além do nível cultural da população e da forma de manejo da terra e do alimento ${ }^{17}$.

Neste trabalho não foi verificada a presença de nenhuma estrutura parasitária de protozoários. Esse fato pode ser esclarecido pela lavagem das hortaliças com água antes dos feirantes colocálas à venda ${ }^{21}$. As folhas, em sua maioria, estavam molhadas, possivelmente os vendedores lavaram as alfaces e removeram os protozoários. Por outro lado, a estrutura e tamanho dos helmintos facilita a adesão às hortaliças.

Avaliaram a qualidade microbiológica de alfaces (Lactuca sativa) comercializadas em supermercados e feiras livres na cidade de Recife, Pernambuco, e demonstraram que $100 \%$ das amostras analisadas estavam contaminadas por coliformes totais e $100 \%$ por coliformes termotolerantes, o que, segundo os autores, representa a situação precária da higiene sanitária do alimento, pois a detecção de 
coliformes a 45 ㅇ C indica a contaminação fecal dessa hortaliça ${ }^{22}$.

Pesquisa realizada em Sorocaba, São Paulo, sobre as condições higiênico-sanitárias das verduras e legumes comercializados pela Companhia de Entrepostos e Armazéns Gerais de São Paulo (CEAGESP), verificaram a presença de coliformes termotolerantes em $87 \%$ das amostras, o que indica falha na higienização e falta de saneamento básico ${ }^{23}$.

As feiras livres representam uma forma de comércio popular, onde os produtores rurais vendem suas hortaliças, na maioria das vezes, como forma de sustento familiar ${ }^{8}$. A busca por alimentos que ofertem benefícios à saúde e que sejam isentos de substâncias que podem acarretar, a curto ou longo prazo, danos ao organismo, fez com que o consumidor optasse pelo consumo de alimentos provenientes da agricultura orgânica, na qual não se utiliza os agrotóxicos ${ }^{24}$. Porém, há alguns questionamentos a respeito do risco mais elevado de contágio microbiológico e parasitário nos alimentos originados pelo sistema orgânico em virtude do uso de dejetos fecais animais e humanos ${ }^{25}$.

Sendo assim, a presença de coliformes termotolerantes está diretamente relacionada com a falha na higiene, que pode ocorrer desde o plantio até a comercialização da hortaliça, causando riscos para a saúde do consumidor, visto que, diversas doenças podem ser causadas pela ingestão de coliformes fecais ${ }^{7}$.

Segundo Souza ${ }^{26}$, infecções causadas pelos coliformes termotolerantes, em que a protagonista é a bactéria Escherichia coli, podem se disseminar pelo organismo causando meningites e infecções gastrointestinais. Ainda de acordo com o autor, de 15 a $20 \%$ das crianças nos seus primeiros anos de vida são acometidas por diarreias causadas pelos coliformes termotolerantes. Contudo, a principal forma de se evitar essas e outras doenças geradas por esses microrganismos é através da higienização dos alimentos.

Sendo assim, através do processo de higienização, apenas com água corrente, ocorre uma redução no número de amostras contaminadas por microrganismos em torno de $42 \%$ e $50 \%$, para coliformes totais e termotolerantes, respectivamente. Com a utilização do hipoclorito de sódio há a eliminação dessas bactérias em $100 \%$ das amostras ${ }^{27}$.

É de extrema importância que a correta higienização seja realizada previamente ao consumo de alimentos in natura, a fim de impedir a disseminação de diversos patógenos e o desenvolvimento de doenças. De preferência, a higienização da hortaliça deve ser feita imediatamente após a aquisição, a limpeza deve ser realizada com água corrente na superfície do alimento e deve-se retirar as partes danificadas. Posteriormente, deve-se utilizar o hipoclorito de sódio dissolvido em água, uma vez que a limpeza realizada somente com água, ou o uso de ácido acético não são eficientes. O recipiente utilizado para o armazenamento deve estar limpo e fechado impedindo o contato com o $\operatorname{ar}^{14}$.

\section{Considerações finais}

A presença de parasitos e coliformes termotolerantes nas amostras de alfaces ( $L$. sativa) obtidas em feiras livres no município de Jequié-Bahia indica que as condições de higienização são precárias na produção, armazenamento, manipulação e/ou transporte desta hortaliça. A falta de saneamento básico e do tratamento da água utilizada na irrigação, também são fatores que corroboram para a contaminação das hortaliças. Dessa forma tornase imprescindível orientar produtores e consumidores quanto às medidas corretas para higienização desta e de outras hortaliças, a fim de que evite a transmissão de doenças veiculadas por alimentos.

\section{Referências}

1. Alves JA, Boas EVdBV, Boas BMV, Souza ÉCd. Qualidade de produto minimamente processado à base de abóbora, cenoura, chuchu e mandioquinha-salsa. Ciência e Tecnologia de Alimentos. 2010; 30(3): p. 625-634.

2. Abougrain AK, Nahaisi MH, Madi NS, Saieb MM, Ghenghesh KS. Parasitological contamination in salad vegetables in Tripoli-Liby. Food Control. 2010; 21:760-762.

3. Adanir R; Tasci F. Prevalence of helminth eggs in raw vegetables consumed in Burdur, Turkey. Food Control. 2013;131: 482-484.

4. Ezatpour B, Chegeni AS, Abdollahpour F, Aazami M, Alirezaei M. Prevalence of parasitic contamination of raw vegetables in Khorramabad, Iran. Food Control. 2013; 34:92-95.

5. IBGE. Instituto Brasileiro de Geografia e Estatística. Munic: mais da metade dos municípios brasileiros não tinha plano de saneamento básico em 2017. Disponível em: https://agenciadenoticias.ibge.gov.br/agenciasala-de imprensa/2013-agencia- 
denoticias/releases/22611-munic-mais-dametade-dos-municipios-brasileiros-não-tinhaplano-de-saneamento-basico-em-2017

6. Vollkopf PCP, Lopes FMR, Navarro IT. Ocorrência de enteroparasitos em amostras de alface (Lactuca sativa) Comercializadas em Porto Murtinho-MS. Arq. ciên. vet. zool. UNIPAR, Umuarama.2006;9 (1):38-40.

7. Ferreira MB, R.Nespolo C, Brasil CCB, Bordin LC, Teixeira BK, Model BP, et al. Avaliação microbiológica de hortaliças folhosas na região Fronteira Oeste do Rio Grande do Sul, Brasil. Associação Argentina de Horticultura. 2018; p. 23-34.

8. Soares B, Cantos GA. Qualidade parasitológica e condições higiênico-sanitárias de hortaliças comercializadas na cidade de Florianópolis, Santa Catarina, Brasil. Rev. Bras.Epidemiol. 2005;8(4):377-84.

9. Rocha A, Mendes RA, Barbosa CS. Strongyloides spp e outros parasitos encontrados em alfaces (Lactuca sativa) comercializados na cidade do Recife, PE. Revista de Patologia Tropical. 2008;37(2):151-160.

10. Rosa CCB, Martins MLL, Folly MM. Avaliação microbiológica de hortaliças provenientes de hortas comunitárias de Campos dos Goytacazes, RJ. Higiene Alimentar. 2005; 19(134): p. 75-80.

11. Silva N, Junqueira VCA, Silveira NFA. Manual de Métodos de Análises Microbiológicas de Alimentos. São Paulo: Varela, 1997.

12. Brasil, 2001. Agência Nacional de Vigilância Sanitária. [Online]; 2001. Acesso 23 de Agosto de 2019. Disponivel em: http://portal.anvisa.gov.br/documents/33880/25 68070/RDC 12 2001.pdf/15ffddf6-3767-4527bfac-740a0400829b

13. Santos NM, Sales EM, Santos $A B$, Damasceno KA, Thé TS. Avaliação parasitológica de hortaliças comercializadas em supermercados e feiras livres no município de Salvador/Ba.Rev.ci.méd.biol.2009;8(2):146-152.

14. Gregório DS, Moraes GFA, Nassif JM, Alves MRM, Carmo NE, Jarrouge MG, et al. Estudo da contaminação por parasitas em hortaliças da região leste de São Paulo. Science in Health. 2012; 3(2):96-103.

15. Brasil, 1978. Comissão Nacional de Normas e Padrões para Alimentos-CNNPA/ANVISAAgência nacional da Vigilância Sanitária. Normas técnicas especiais, no 12, de 1978. São Paulo: CNNPA/ ANVISA; 1978.

16. Pacifico BB, Bastos OMP, Uchôa CMA.
Contaminação parasitária em alfaces crespas (Lactuca sativa var. crispa), de cultivos tradicional e hidropônico, comercializadas em feiras livres do Rio de Janeiro (RJ). Rev. Inst. Adolfo Lutz. 2013;72(3):219-225.

17. Alves AS, Neto AC, Rossignoli PA. Parasitos em alface-crespa (Lactuca sativa I.), de plantio convencional, comercializada em supermercados de Cuiabá, Mato Grosso, Brasil. Revista Patologia Tropical. 2013;42(2):217-229.

18. França BR, Bonnas DS, Silva CMO. Qualidade higiênico sanitária de alfaces (Lactuca sativa) comercializadas em feiras livres na cidade de Uberlândia, MG, Brasil. Biosci.J. 2014;30(1):458-466.

19. Normura PR, Ferreira ARM, Rafaelli RA, Augusto JG, Tatakihara VLH, Custódio LA, et al. Estudo da incidência de parasitas intestinais em verduras comercializadas em feira livre e supermercado de Londrina. Semina: Ciências Biológicas e da Saúde.2015;36(1):209-214.

20. Oliveira CAF, Germano PML. Estudo da ocorrência de enteroparasitas em hortaliças comercializadas na região metropolitana de São Paulo, SP, Brasil, I - Pesquisa de helmintos. Revista Saúde Pública. 1992;26(4):283-289.

21. Falavigna LM, Freitas CBR, Melo GC, Nisshi L, Araújo SM, Guilherme-Falavigna AL. Qualidade de hortaliças comercializadas no noroeste do Paraná, Brasil. Parasitol Latinoam. 2005; 60:144149.

22. Shinohara NKS, Lima TBN, Siqueira LP, Pereira JAP, Padilha MR. Avaliação da qualidade microbiológica de alfaces (Lactuca sativa) comercializadas em feiras livres e supermercados do Recife, Brasil. Diálogos Acadêmicos. 2014; 6(1).

23. Pacheco MAdSR, Fonseca YSK, Dias HGG, Candido, VLP, Gomes, AHdS, Armelin, IM, Bernardes R. Condições higiênico-sanitárias de verduras e legumes comercializados no Ceagesp de Sorocaba - SP. Revista Higiene Alimentar. 2002; 16(101): p. 50-55.

24. Sediyama MAN, Santos IC, Lima PC. Cultivo de hortaliças no sistema orgânico. Revista Ceres. 2014; 61:829-837.

25. Arbos KA, Freitas RJS, Stertz SC, Carvalho LA. Segurança alimentar de hortaliças orgânicas: aspectos sanitários e nutricionais. Ciência e Tecnologia de Alimentos. 2010; 30:215-220.

26. Souza, CP. Segurança alimentar e doenças veiculadas por alimentos: utilização do grupo coliforme como um dos indicadores de qualidade de alimentos. Revista APS. 2006; 9(1): p. 83-88. 
27. Borco SE, Pierozan MK, Cansian RL, Oliveira Dd, Pinheiro TdLF, Toniazzo G. Condições higiênicas de alfaces (Lactuca sativa) comercializadas na cidade de Erechim- RS. Revista Brasileira de Alimentos e Nutrição / Alimentos e Nutrição. 2011: p. 301-305.

\section{Endereço para Correspondência}

Dellane Tigre

Rua Carlos Caires Brito

E-mail: dellanetigre@yahoo.com.br

Recebido em 05/07/2021

Aprovado em 08/12/2021

Publicado em 30/12/2021 